Preprint typeset in JHEP style. - HYPER VERSION

SUGP-01/2-1

hep-th/0103098

\title{
T-duality and the case of the disappearing brane
}

\author{
Donald Marolf \\ Physics Department, Syracuse University, Syracuse, New York 13244
}

\begin{abstract}
When an NS5-brane crosses a D6-brane, a D4-brane is created stretching between them by the Hanany-Witten effect. However, the T-dual situation involves a Kaluza-Klein monopole crossing a D5-brane and should not result in brane creation. Thus, the newly created D4-brane disappears when T-duality is applied. The T-duality is in a direction transverse to the D4-brane so that one would naively have expected the creation of a D5-brane in the T-dual picture. This situation is investigated via the corresponding supergravity solutions and the tension with the naive result is resolved. Along the way, a differential form version of the supergravity T-duality relations is developed and some comments are made concerning the flux-expulsion properties of D6-branes.
\end{abstract}

Keywords: Branes, Brane Junctions, M-theory, Kaluza-Klein Monopoles. 


\section{Contents}

1. Introduction 1

2. The smooth picture of the Hanany-Witten effect 2

3. The IIB description 6

3.1 A more geometric form of the T-duality relations 6

3.2 Surfaces, Charges, and disappearing branes 8

4. Discussion 11

\section{Introduction}

In the last ten years it has come to be widely recognized that dualities are one of the fundamental structures of string- and M-theory. T-duality, which interchanges small circles with large ones, was one of the first dualities to be discovered and understood, see e.g. [1] for a review. The familiar result that applying T-duality in a direction orthogonal to a Dp-brane results in a $\mathrm{D}(\mathrm{p}+1)$-brane (and similarly that T-duality applied along a Dp-brane results in a $\mathrm{D}(\mathrm{p}-1)$-brane) is widely used to relate properties of the various Dp-branes. It is therefore of interest to uncover any cases which deviate from this rule and to carefully understand how such deviations occur.

The situation analyzed below is one to which precise application of the above rule connecting $\mathrm{Dp}$ - and $\mathrm{D}(\mathrm{p} \pm 1)$-branes by $\mathrm{T}$-duality would raise a contradiction. We consider the case of a D4-brane created via the Hanany-Witten effect by the crossing of a D6-brane and an NS5-brane. The two branes have three spatial directions in common but are otherwise orthogonal. The D4-brane also lies along the three common directions but its fourth direction is orthogonal to both the NS5 and D6-branes, since the D4-brane stretches between them. This setting is summarized by the table below, where the X's indicate directions along the indicated brane.

\begin{tabular}{|c|c|c|c|c|c|c|c|c|c|}
\hline & $x_{1}$ & $x_{2}$ & $x_{3}$ & $x_{4}$ & $x_{5}$ & $x_{6}$ & $x_{7}$ & $x_{8}$ & $x_{9}$ \\
\hline $\mathrm{D} 6$ & $\mathrm{X}$ & $\mathrm{X}$ & $\mathrm{X}$ & $\mathrm{X}$ & $\mathrm{X}$ & $\mathrm{X}$ & & & \\
\hline $\mathrm{NS} 5$ & $\mathrm{X}$ & $\mathrm{X}$ & $\mathrm{X}$ & & & & $\mathrm{X}$ & $\mathrm{X}$ & \\
\hline $\mathrm{D} 4$ & $\mathrm{X}$ & $\mathrm{X}$ & $\mathrm{X}$ & & & & & & $\mathrm{X}$ \\
\hline
\end{tabular}


In the low energy regime, the Hanany-Witten effect [2] can be understood from either the worldvolume (Born-Infeld-Chern-Simons) theories of the branes [3] or from a spacetime perspective in supergravity [4 as well as from a number of more stringy arguments [5, 6, 0, 8, 9, 10, 11. We find the supergravity perspective particularly useful in analyzing this situation and, as is discussed below, this analysis clearly shows the creation of the D4-brane. Such solutions were constructed and analyzed in [12 based on the work of [13, 14]. Our setting is related to the more familiar creation of a fundamental string by the crossing of D3 and D5-branes (see e.g. [15]) by T-duality along the three directions common to the NS5 and D6-branes and a final S-duality transformation in the type IIB theory.

Consider, however, the application of T-duality in a direction along the D6-brane but orthogonal to the NS5 and D4-branes (say along $x_{4}$ ). This turns the NS5-brane into a Kaluza-Klein monopole, so the IIB version is the crossing of a D5-brane and a Kaluza-Klein monopole. Naively, T-duality would turn the D4-brane into a D5-brane. However, one can show that D5-branes cannot be created by the crossing of KaluzaKlein monopoles and other D5-branes. In fact, D5-branes can be created only in the presence of NS5-branes or D7-branes.

We find a resolution to this puzzle in the structure of the Kaluza-Klein monopole. The T-duality that creates the Kaluza-Klein monopole involves a certain cutting and pasting of the original spacetime with the property that closed surfaces in the original type IIA spacetime are not naturally mapped to closed surfaces in the final IIB spacetime. Ignoring this fact would lead to the naive result, involving creation of D5-branes and a contradiction. However, a more careful treatment shows that no net D5 charge is created and no Hanany-Witten effect takes place. We analyze the situation using the corresponding supergravity solutions.

The plan of the paper is as follows. We begin with a brief review in section 2 of the picture of the smooth creation of branes via the Hanany-Witten effect in supergravity. It will be clear from this discussion that the IIB intersection T-dual to the IIA D4-brane ending on the D6-brane cannot be merely a D5-brane three-brane junction as one might otherwise expect. In section 3, we use a differential form version of the supergravity T-duality relations to map to the IIB setting, to evaluate the various charges, and to illustrate the connection to the closed surfaces mentioned above. Finally, we end with some discussion in section 4 , including comments relating D6-branes to "superconducting black holes" [16].

\section{The smooth picture of the Hanany-Witten effect}

This section provides a brief review of how the Hanany-Witten brane creation effect 
is described as a smooth process. The basic picture follows from general principles, but one can also find a one-parameter moduli space of exact BPS solutions describing certain versions of the process in either the worldvolume theory of a D-brane in the background generated by another D-brane [15] or in full supergravity [12, 4]. What happens in either the worldvolume theory or supergravity is that the flux of a gauge field generated by one brane falling on the second brane generates a third kind of charge associated with the new brane.

In the supergravity description, this effect follows from the fact that the 'branesource' charge of the D4-brane (see [4]) is not conserved [12, 团. This in turn is a straightforward consequence of the modified Bianchi identity satisfied by the gauge invariant Ramond-Ramond four-form field strength $\tilde{F}_{4}$ of which the D4-brane is a magnetic source. We have the relation

$$
d \tilde{F}_{4}+F_{2} \wedge H_{3}=* j_{D 4}^{b s},
$$

where the right hand side is the brane-source current (which vanishes in the absence of an explicit D4-brane source). Here, $F_{2}$ is the usual IIA Ramond-Ramond two-form field strength and $H_{3}=d B_{2}$ is the Neveu-Schwarz field strength. Taking an exterior derivative of $(2.1)$ shows that $d * j_{D 4}^{b s}$ does not vanish. Instead, a flux of $F_{2}$ falling on an NS5 brane (where $* j_{N S 5}^{b s} \equiv d H_{3} \neq 0$ ) or a flux of $H_{3}$ falling on a D6-brane (where $\left.* j_{D 6}^{b s} \equiv d F_{2} \neq 0\right)$ acts as a source or sink of D4-brane charge. Some of the subtleties of defining charge and working with brane-source currents are discussed in [4], but it is enough for us that this result leads to the Hanany-Witten effect and the associated creation of a D4-brane as described below.

The diagram below shows various stages in this process for the case of an NS5brane moving past a D6-brane to make a D4-brane [12]. Similar results also follow for $\mathrm{D} p$ and $\mathrm{D} p^{\prime}$ branes whenever $p+p^{\prime}=8$, see e.g. 115 for a worldvolume description of the D3/D5 case. At stage (i) when the NS5-brane is far from the D6-brane, the center of the NS5-brane subtends a small angle at the D6-brane and captures only a small amount of flux from the D6. As a result, essentially no D4 charge is induced in the region shown and one has only a flat NS5-brane. Then, as the NS5-brane approaches the D6-brane (ii), it subtends a larger angle and begins to capture some flux, generating some D4 charge. This charge corresponds to D4-branes lying inside the NS5-brane and running outward along this brane to infinity.

When the NS5-brane is dragged past the D6-brane (iii), all of the flux from the D6-brane is captured in the part of the NS5-brane close to the D6-brane. Capturing one quantum of flux corresponds to the creation of one quantum of fundamental string charge, so that the thin neck of NS5-brane approximates a single D4-brane. However, the NS5-brane captures flux of the opposite sign in the region where the neck joins the 


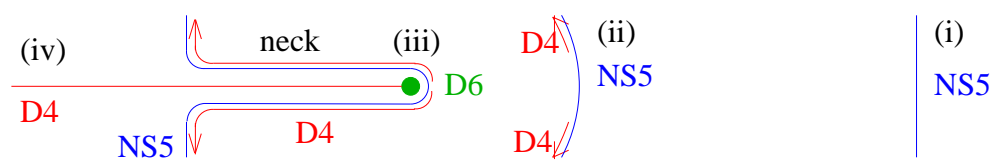

Figure 1: Four stages in the Hanany-Witten process.

asymptotically flat part of the NS5-brane. The flux captured in this region is half of that generated by the D6-brane, so that a net one-half quantum of D4 charge reaches infinity along the NS5-brane. This last statement is true in each of the stages (i,ii,iii,iv), though only in stage (iii) are all of the relevant parts of the NS5-brane visible in figure 1. In stage (iv), the neck has narrowed so as to become difficult to resolve and all that remains is a D4-brane string stretching between an NS5-brane and a D6-brane.

One odd feature of the above discussion is that nowhere have we mentioned the creation of D4-branes by $\mathrm{H}_{3}$ flux falling on the D6-brane. This is in fact the correct result, as may be seen from the explicit exact solutions in [12]. It is related to a 'fluxexpulsion' effect associated with D6-branes and reminiscent of the 'superconducting branes' phenomenon [16]. We will discuss this effect and related issues further in section 4

Each stage in figure 1 depicts a BPS configuration of either the worldvolume theory (as described in [15]) or supergravity (following [12]). Studying BPS configurations is useful for a variety of well-known reasons. However, one should also apply the lessons learned from BPS configurations to non-BPS contexts. For example, we learn from the above diagram that there is no fundamental difference between a D4-brane ending on a D6-brane and an NS5-brane tightly wrapped around the D6-brane. Indeed, we would generically expect fluctuations (dynamical, thermal, or quantum mechanical) between such configurations. For the case above, both configurations are BPS. However, this is a function of the detailed boundary conditions: note that the asymptotics ${ }^{1}$ of the NS5-brane are different at stages (i) and (iv).

If one fixes the boundary conditions then there is only one BPS configuration and any fluctuations should cost energy. In particular, suppose that we fix the boundary conditions to be those of stage (iv). We then conclude that there are fluctuations in which the attachment of the D4 to the D6 'loosens' into the configuration shown below ${ }^{2}$.

\footnotetext{
${ }^{1}$ In both cases, the asymptotics should be those of a logarithmic curve [17] due to the fact that the end of the D4-brane in the NS5 is of co-dimension 2. The case of a D2-brane crossing a D6-brane and creating a fundamental string is very much the same. See [18] for some interesting comments on the relation of this logarithmic curve to the fundamental string charge flowing through the D2-brane. These comments also apply to the current NS5/D6/D4 case.

${ }^{2}$ Again, the same effect arises in a D2/D6/F1 system, with the fluctuations now being between a tightly attached fundamental string and one loosely attached by a loop of D2-brane. One has to wonder
} 
However, due to the tension of the NS5-brane, this loosely attached configuration has more energy than the tightly attached configuration and the 'noose' will tighten of its own accord. In particular, in the limit in which string perturbation theory becomes valid $\left(g \rightarrow 0\right.$ with $\ell_{s}$ fixed) the NS5-brane (whose tension is proportional to $1 / g^{2}$ ) becomes very heavy relative to a D4-brane (with tension $T_{D 4} \sim 1 / g$ ) and the energy difference between the loosely and tightly attached configurations diverges. This is of course consistent with the usual observation that only the tightly attached configuration (of either D4's or F1's ending on D6's) is relevant in perturbation theory and that one sees no sign there of fluctuations to a loosely attached configuration.



Figure 2: A D4-brane 'loosely attached' to D6-brane.

The above discussion followed directly from an analysis of D4 brane-source charge conservation. Let us now consider a T-dual configuration, where the T-duality is applied in a direction along the D6-brane but orthogonal to the NS5-brane. This direction will also be orthogonal to the D4-brane created above.

It is simplest to begin with stage (i), which contains only two widely separated branes. The T-dual configuration clearly contains a D5-brane and a Kaluza-Klein monopole, which is the T-dual of the NS5-brane. To see whether a D5-brane can be created in stages (ii) and (iii), we should analyze conservation of the D5 brane-source charge. The associated gauge invariant field strength $\tilde{F}_{3}$ does satisfy a modified Bianchi identity, but in the form

$$
d \tilde{F}_{3}-F_{1} \wedge H_{3}=* j_{D 5}^{b s}
$$

where $F_{1}$ is the IIB one-form field strength associated with D7-branes. Here our conventions for the IIB fields differ from those of [20] by a sign, in order that we make a choice compatible with the conventions of [21, 22, 23]. Taking an exterior derivative, we see that $* j_{D 5}^{b s}$ is conserved except perhaps at D7-branes or NS5-branes - neither of which appear in our setting. As a result, D5 brane-source charge is conserved and no creation of D5-branes can occur ${ }^{3}$.

about the implications of this observation for understanding black hole entropy non-perturbatively. Naive extrapolation suggests that at finite $g$ (and finite $N$ ) the states counted by Strominger and Vafa 19] are not well localized on D-branes. Perhaps this is related to the fact that black hole solutions of supergravity have interiors of finite size?

${ }^{3}$ To add further weight to the argument it is useful to notice that one could further S-dualize our IIB setting and obtain a pure NS solution involving an NS5-brane and a Kaluza-Klein monopole. One 
Considering the ending of the D4-brane on the D6-brane, one might have thought that the T-dual description should be a three D5-brane junction, which does indeed conserve D5-brane charge: the charge simply flows from one brane to the other. However, this option is ruled out by the fact that on the IIA side the D4-brane is smoothly created before the NS5 and D6-branes come into contact. As a result, there is no way for D5-brane charge to flow between the branes in stages (ii) and (iii) above.

\section{The IIB description}

In this section, we analyze the charges of the T-dual IIB spacetime. Luckily, we will find that one need not explicitly write down the IIB solution. Instead, we will be able to evaluate the various charges directly from our knowledge of the IIA case.

The charge in which we are particularly interested is the D5-charge of the created brane. Since the D5-charge is conserved in this setting, such charges can best be computed by integrating the flux of $\tilde{F}_{3}$ over an appropriate sphere. It is therefore useful to work with the T-duality relations between IIA and IIB supergravity written in a geometric way associated with differential forms instead of the original component descriptions used in [21, 22]. We therefore briefly reformulate these relations in subsection 3.1 before moving on to study the IIB solution in subsection 3.2.

\subsection{A more geometric form of the T-duality relations}

One can check that the results given in [21] for the action of T-duality on NeveuSchwarz fields and in 22] for the action on Ramond-Ramond fields are equivalent to the following description of T-duality in IIA/IIB supergravity. Let us begin with a spacetime having a Killing field $\lambda$ with $S^{1}$ orbits. Let $\tilde{\lambda}_{\mu}=\lambda_{\mu} /|\lambda|^{2}$ denote the oneform obtained by lowering the index on the Killing field $\lambda$ with the string metric and dividing by its norm. Let $y$ denote any coordinate along the orbits of $\lambda$ satisfying $\lambda^{\mu} \partial_{\mu} y=1$. We normalize the Killing field by requiring $y$ to have period $2 \pi \ell_{s}$, where $\ell_{s}$ is the string length. Thus, for a spacetime that may be treated classically $|\lambda|$ will be of size $R / \ell_{s}$ for some macroscopic length scale $R$.

Just as in Kaluza-Klein reduction, one can split the metric into a part $\left(d s_{r}^{2}\right.$, the reduced metric) associated with intervals transverse to the orbits of $\lambda$ and a part along $\tilde{\lambda}$ :

$$
d s_{\text {string }}^{2}=d s_{r}^{2}+|\lambda|^{2} \tilde{\lambda} \otimes \tilde{\lambda}
$$

is then free to reinterpret this as a IIA solution and lift to eleven dimensions. In that context, the created brane would be an M5-brane. But the M-theory 4-form field strength satisfies an unmodified Bianchi identity and M5 brane-source charge is conserved in all settings. 
Similarly, the Neveu-Schwarz B-field can be reconstructed from the one-form $(B \cdot \lambda)_{\nu}=$ $B_{\nu \mu} \lambda^{\mu}$ and the two-form $B^{r}=B-\frac{1}{2}(d y+B \cdot \lambda) \wedge \tilde{\lambda}$. Introducing the general notation $\left(C_{n} \cdot \lambda\right)_{\alpha_{1} \ldots \alpha_{n-1}}=\left(C_{n}\right)_{\alpha_{1} \ldots \alpha_{n-1} \alpha_{n}} \lambda^{\alpha_{n}}$ for an $\mathrm{n}$-form $C_{n}$, the reader may check that the T-duality relations of [21, 22] may be written:

$$
\begin{aligned}
\widehat{\phi} & =\phi-\ln (|\lambda|) \\
\widehat{d s}_{r}^{2} & =d s_{r}^{2} \\
\widehat{B}_{x} & =B_{r} \\
\tilde{\tilde{\lambda}} & =d y+B \cdot \lambda \\
d y+\widehat{B} \cdot \lambda & =\tilde{\lambda} \\
|\widehat{\lambda}|^{2} & =\frac{1}{|\lambda|^{2}} \\
\widehat{C}_{n} & =C_{n+1} \cdot \lambda+\left(C_{n-1}-C_{n-1} \cdot \lambda \wedge \tilde{\lambda}\right) \wedge(d y+B \cdot \lambda),
\end{aligned}
$$

where hatted symbols (e.g. $\hat{\tilde{\lambda}}$ ) denote fields in the T-dual solution and $C_{n}$ are the Ramond-Ramond gauge potentials. The understanding in (3.2) is that the two solutions are thought of as living on the same manifold ${ }^{4}$ with $\lambda$ representing the same vector field in each case. However, after performing the T-duality it may be natural to extend the manifold to a larger one that is geodesically complete, e.g. in the duality between the NS5-brane and the Kaluza-Klein monopole in which the fixed points of the Killing field must be added by hand after applying (3.2). Note that although $\lambda^{\mu}$ represents the same vector field on both the IIA and IIB sides, the one-forms $\tilde{\lambda}_{\nu}=\frac{\lambda^{\mu} g_{\mu \nu}}{\lambda^{\alpha} g_{\alpha \beta} \lambda^{\beta}}$ and $\widetilde{\tilde{\lambda}}_{\nu}=\frac{\lambda^{\mu} \hat{g}_{\mu \nu}}{\lambda^{\alpha} \hat{g}_{\alpha \beta} \lambda^{\beta}}$ differ due to changes in the metric.

Since T-duality is properly applied when the orbits of the Killing vector field are compact, one should think of these relations as holding on a circle bundle over the reduced space of orbits. Note that (3.2) is not completely geometric due to the fact that T-duality mixes the metric and Neveu-Schwarz B-field and thus mixes diffeomorphisms with NS gauge transformations. It is for this reason that a choice of coordinate $y$ must be made before one can write down the above relations. Of course, two different choices of $y$ result in spacetimes that differ only by a Neveu-Schwarz gauge transformation.

What we will need most to compute charges is not the potentials given in (3.2) above, but the associated field strengths. If we can choose a gauge in which the Lie derivatives of the potentials $C_{n}, B$ vanish along the Killing field $\lambda$, then the relations $d\left(C_{n} \cdot \lambda\right)=\left(d C_{n}\right) \cdot \lambda$ hold and the field strengths $F_{n}=d C_{n-1}$ satisfy

\footnotetext{
${ }^{4}$ This manifold should consist only of those points where both the metric and the NS gauge potential $B$ are non-singular.
} 


$$
\begin{aligned}
\widehat{F}_{n} & =F_{n+1} \cdot \lambda+\left(F_{n-1}-F_{n-1} \cdot \lambda \wedge \tilde{\lambda}+(-1)^{(n-2)} C_{n-2} \cdot \lambda \wedge d \tilde{\lambda}\right) \wedge(d y+B \cdot \lambda) \\
& +(-1)^{n-2}\left(C_{n-2}-C_{n-2} \cdot \lambda \wedge \tilde{\lambda}\right) \wedge H \cdot \lambda
\end{aligned}
$$

where $H=d B$. Of course, not all of these field strengths are gauge invariant. In particular, neither the field strength $F_{4}=d C_{3}$ which couples magnetically to D4branes nor the $F_{3}=d C_{2}$ which couples magnetically to D5-branes is gauge invariant. Instead, the gauge invariant versions $\operatorname{are}^{5}$

$$
\begin{aligned}
& \tilde{F}_{3}=F_{3}+C_{0} \wedge H, \\
& \tilde{F}_{4}=F_{4}-C_{1} \wedge H .
\end{aligned}
$$

It follows from (3.3) above that these are related by

$$
\widehat{\tilde{F}}_{3}=\tilde{F}_{4} \cdot \lambda+\left(F_{2}-F_{2} \cdot \lambda \wedge \tilde{\lambda}\right) \wedge(d y+B \cdot \lambda) .
$$

Taking a further exterior derivative leads to the conclusion that the D4, D5, and D6 brane-source charges satisfy

$$
* j_{D 5}^{b s}=* j_{D 4}^{b s} \cdot \lambda+\left(* j_{D 6}^{b s}-* j_{D 6}^{b s} \cdot \lambda \wedge \tilde{\lambda}\right) \wedge(d y+B \cdot \lambda) .
$$

Similar relations hold for the other D-branes.

As a result, at least if the branes appear in a part of the spacetime where the circle bundles are smooth, the T-dual of a D4 brane-source current is indeed a D5 brane-source current when the T-duality acts orthogonally to the D4-brane.

\subsection{Surfaces, Charges, and disappearing branes}

From the results of the last subsection, one would be tempted to conclude that our D4-branes are replaced by D5-branes in the T-dual type IIB spacetime. However, the subtlety is that these branes would lie exactly at the points where the circle bundle description breaks down. In particular, on the the IIB side they would lie at the core of the Kaluza-Klein monopole where the circles degenerate to a point ${ }^{6}$. As a result, we must compute D5-brane charge by considering flux integrals of $\tilde{F}_{3}$ over various surfaces in the spacetime. Since $\lambda$ is along the D6-brane, one expects $C_{1} \cdot \lambda$ and $F_{2} \cdot \lambda$ to vanish

\footnotetext{
${ }^{5}$ Again, our conventions are compatible with [21, 22, 23. However, while they agree with those of 20 on the IIA side, they differ with 20] by a sign on the IIB side.

${ }^{6}$ Singularities associated with branes can sometimes be removed by smearing out the charge over a finite volume. However, there is no smooth spacetime corresponding to a smeared Kaluza-Klein monopole just as there is no fiber bundle that realizes the idea of a smeared magnetic charge.
} 
on the IIA side. This may be checked explicitly using the solution of [12]. One may also check that the Lie derivatives along $\lambda$ of $C_{n}$ and $B$ vanish. As a result, equation (3.5) becomes just

$$
\begin{aligned}
\widehat{\tilde{F}}_{3} & =F_{4} \cdot \lambda-C_{1} \wedge H \cdot \lambda+F_{2} \wedge(d y+B \cdot \lambda) \\
& =d\left[C_{3} \cdot \lambda+C_{1} \wedge(d y+B \cdot \lambda)\right] .
\end{aligned}
$$

In particular, $\widehat{\tilde{F}}_{3}$ is a total derivative, as one would expect in a situation in which the associated D5-brane charge is conserved. As a result, integrals of this field over a closed surface can be evaluated purely in terms of contributions from submanifolds where the potentials are ill-defined; i.e., from Dirac strings. It is useful to note that our D5 charge can be evaluated from the Dirac strings of the associated type IIA fields $C_{3}, C_{1}$, and $B$.

Before proceeding, we pause briefly to acquaint the reader with the coordinate system used in [12]. The Cartesian coordinates $x_{0}$ (time), and $x_{1}, x_{2}, x_{3}$ are taken to run along all three branes (D6, NS5, and D4). The T-duality will be performed along the $x_{4}$ direction, which is also along the D6brane. Here consider the D4- and D5-branes to be smeared along this direction in order to generate the required translational symmetry. The coordinates $x_{5}, x_{6}$ are along the D6brane but transverse to the NS5- and D4-branes.

For the rest of the coordinate system, we take $x_{7}, x_{8}, x_{9}$ to be Cartesian-like coordinates in the space transverse to the

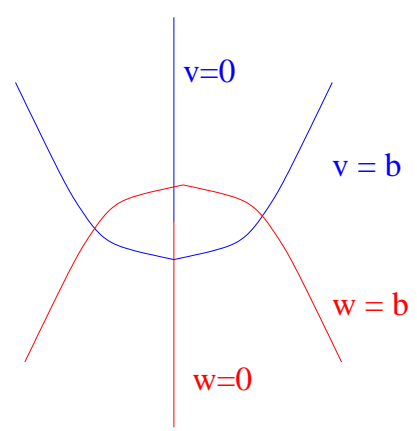

Figure 3: The v,w coordinates. D6-brane which are defined in a manner adapted to the spherical symmetry of the $F_{2}$ field. When the NS5-brane is far from the D6-brane (i.e., stage (i)), we may think of it as lying in the $x_{7}, x_{8}$ directions at $x_{9}=$ constant. However, when the NS5-brane approaches the D6-brane it deforms and is no longer flat. As a result, to describe stages (ii) through (iv) it is useful to introduce the coordinates $v, w$ through

$$
\begin{aligned}
r^{2} & =x_{7}^{2}+x_{8}^{2}+x_{9}^{2}, \\
\cos \theta & =x_{9} / r \\
v & =r \sin \theta / 2, \\
w & =r \cos \theta / 2,
\end{aligned}
$$

In particular, in the region close to the D6-brane core the NS5-brane lies on the surface $v=$ constant . 
We are interested in the following surfaces. First, we wish to measure the total D5-brane charge flowing down from infinity along the Kaluza-Klein monopole. So, we need a 3 -sphere surrounding the $v=0$ axis (and in fact outside the surface $\left.v=b, x_{5}=x_{6}=0\right)$. One particular choice in the IIA near D6-brane spacetime would be the surface $S_{U}$ ( $U$ for upper) defined by

$$
\begin{array}{r}
v^{2}+w^{2} \equiv r^{2}=\text { const } \\
v^{2}+x_{5}^{2}+x_{6}^{2} \equiv R_{U}^{2}=\text { const } \\
x_{0}, x_{1}, x_{2}, x_{3}, x_{4}=0 .
\end{array}
$$

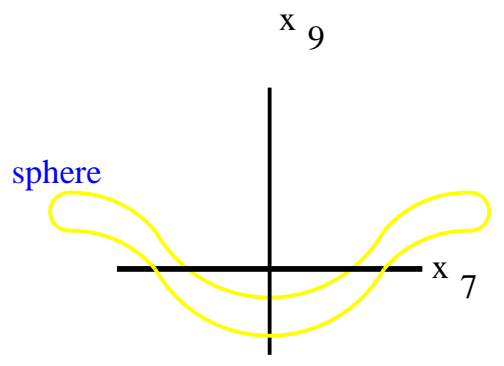

Figure 4: The upper sphere.

This three-sphere lies above the second brane and surrounds the monopole so long as $r^{2}>2 R_{U}^{2}$ and $R_{U}>b$. A 'side view' of this sphere is shown below, where the $x_{7}$ and $x_{9}$ axes are shown and the direction out of the page represents, say, $x_{6}$. The closer side of the sphere passes in front of the $x_{7}$ and $x_{9}$ axes with the farther size passing behind.

We would also like to measure the charge flowing outward along the second brane. To do so requires a surface surrounding this brane in an appropriate sense. Some thought shows that the correct surface has the topology $S^{2} \times S^{1}$ and that a particular example is the surface $S_{0}$ given by taking

$$
\begin{array}{r}
v^{2}+w^{2} \equiv r^{2}=\text { const }, \\
x_{5}^{2}+x_{6}^{2} \equiv R_{0}^{2}=\text { const } \\
x_{0}, x_{1}, x_{2}, x_{3}, x_{4}=0 .
\end{array}
$$

To double check the results we fill find, we might also consider a surface lying entirely below the second brane. The flux through this sphere should be the sum (with an appropriate sign) of the fluxes through the two surfaces described above. The sphere $S_{L}$ defined by

$$
\begin{array}{r}
v^{2}+w^{2} \equiv r^{2}=\text { const } \\
w^{2}+x_{5}^{2}+x_{6}^{2} \equiv R_{L}^{2}=\text { const } \\
x_{0}, x_{1}, x_{2}, x_{3}, x_{4}=0
\end{array}
$$

lies below the second brane for $r^{2}>2 R_{L}^{2}$.

Let us now consider the Dirac strings of the IIA fields. As in [12, we may we work in a gauge where the Dirac string of $C_{1}$ lies at $v=0$ and does not intersect any of the surfaces above. From [12 one finds that such a gauge has $C_{3}=0$, so that Dirac strings of $C_{3}$ are not a concern. 
What remain are only the IIA Dirac strings of $B$. We pause here to note that these become Dirac strings of the Kaluza-Klein monopole in the IIB solution. At a Dirac string of $B$, one must perform a large gauge transformation in order to smooth out the description of the field. For the Kaluza-Klein monopole, the associated smoothing requires a large coordinate transformation. The result is that if a Dirac string of $B$ intersects a smooth closed surface in the IIA solution defined by coordinate conditions such as those above, then these conditions need not define a smooth closed surface in the IIB solution. This is exactly what happens in the gauge used for $B$ in [12], and performing the associated integrals over our surfaces gives a nonvanishing result for $S_{U}$ and $S_{0}$. The sphere $S_{L}$ does not intersect these strings and so the corresponding flux vanishes. However, due to the Dirac string of the Kaluza-Klein monopole the conditions 3.9,3.10 considered in the IIB solution in this gauge do not in fact define closed surfaces.

As a result, more care must be taken. The simplest way to proceed is to perform a change of gauge in the IIA solution to move the Dirac strings of the B field out of the way. This of course affects neither $C_{1}$ nor $C_{3}$. These Dirac strings can in fact be moved to $x_{5}=x_{6}=0$ and into the region $v<b$ (i.e., inside the paraboloid). To see this, we need only change to $v, w$ coordinates in which the surface $v=b$ becomes a plane. The region $x_{5}=x_{6}=0, v<b$ then becomes the usual half-space shaped Dirac string. In this gauge, the strings do not intersect any of our surfaces $S_{U}, S_{0}$, or $S_{L}$.

Thus, using this new IIA gauge, all three surfaces do in fact represent the closed surfaces we desire in the IIB solution. In addition, the total flux through any of these surfaces vanishes since they intersect no Dirac strings. We conclude that the only D5-branes in the IIB solution are those lying at $v=w=0$.

\section{Discussion}

We have studied a situation in which, instead of transforming into D5-brane charge, D4-brane charge simply disappears under T-duality. Note that in the limit that the IIA NS5-brane degenerates to a line, the IIA configuration appears to be just a D4-brane ending on a D6-brane. On the other hand, the T-dual solution is a D5-brane attached to a strange degenerate configuration of Kaluza-Klein monopole. This latter is some sort of singularity that carries no charge.

There is clearly some tension with the usual result that the T-dual of a braneending configuration is another brane-ending configuration or perhaps a three-brane junction. The resolution appears to stem from the fact that the IIA configuration is a rather subtle type of brane-ending solution. This can be seen by comparing our case with the worldvolume solution of [17] representing a fundamental string ending on a 
D2-brane. One important difference is that in the case of [17 brane-source charge is conserved and flows through the D2-brane from the junction out to infinity. In contrast, it was explicitly shown in [12] that the D4-brane brane-source charge is not conserved in our IIA setting and is directly absorbed by the D6-brane. Indeed, had D4-charge been conserved on the IIA side then it would have been consistent to match this charge to the D5-brane charge on the IIB side (which is certainly conserved). In addition, had D4-brane charge flowed through the D6-brane on the IIA side, one would certainly expect the IIB solution to replace this D6-brane with D5-branes of two sorts: one sort coming from the D4-branes and one from the D6-branes. It would then be quite natural for the IIB solution to be a three-brane junction.

The fact that our solution is not of this type has to do with a somewhat confusing property of D6-branes. Consider any massless type IIA solution containing D6-branes. This of course provides a solution to 11-dimensional supergravity in which the D6branes are replaced by the cores of Kaluza-Klein monopoles. This solution has a Killing field $\lambda^{11}$ which vanishes at the core of each monopole. The natural boundary condition to impose on the D6-branes is that the corresponding 11-dimensional solutions be smooth $^{7}$ at these cores. But now consider the 11-dimensional four-form field strength $F_{4}^{\{11\}}$. If it is smooth then $F_{4}^{\{11\}} \cdot \lambda^{11}$ must vanish when $\lambda^{11}$ does and in particular at any core. Since $H_{3}=F_{4}^{\{11\}} \cdot \lambda^{11}$, it follows that $H_{3}$ will vanish at any D6-brane. Note that since the lowest Fourier mode around the circle will again give some smooth field, this conclusion also holds in cases where the 11-dimensional solution does not have an exact translation symmetry along $\lambda_{11}$ but which can be treated perturbatively. The same argument also applies to the dual field, so that $* \widetilde{F}_{4}=*_{11} F_{4}^{\{11\}}$ should also vanish at a D6-brane. Here $*_{11}$ is the eleven-dimensional Hodge dual. This is the flux that causes D6-branes to produce fundamental strings, so no fundamental string charge should be induced on a D6-brane when a D2-brane is dragged past it.

A similar sort of flux-excluding property was studied in [16. For the 'superconducting' branes considered in that work, the normal component of some field strength was forced to vanish on the horizon. The situation here is somewhat different, however, as now the entire field strength $H_{3}$ or $* \widetilde{F}_{4}$ must vanish at the brane.

The unexpected nature of our IIA solution can be directly traced to this property ${ }^{8}$. Consider again the four stages of section \&. If $H_{3}$ had not vanished at the D6-brane, then the D6-brane would have captured some non-zero flux from the NS5-brane. This

\footnotetext{
${ }^{7}$ In the case of unit-charged monopoles. In the case of multiply-charged Kaluza-Klein monopoles, the natural boundary condition is that an appropriate multiple cover be smooth.

${ }^{8}$ As can the fact that the D6-brane did not deform in space as the NS5-brane was pulled past it, since the deformation of a brane is associated [18] with the tension from the induced charge flowing through the brane to infinity.
} 
would in turn have resulted in the creation of D4-brane charge at the center of the D6brane which would then flow along the D6-brane to infinity. In stages (ii) and (iii) we would see D4-brane charge absorbed on the NS5-brane and created on the D6-brane. However, in the limiting case (iv) the charge would in fact have been conserved as the absorption and creation would both occur at the same place (the intersection). As a result, in this alternate setting one could have said at stage (iv) that the D4 charge flows directly from the NS5-brane to the D6-brane.

Although we have identified the property that leads to our unexpected results and we have shown that this property is a natural result of the eleven-dimensional description, certain aspects of this story remain quite puzzling. For example, flux is clearly not expelled from the NS5-brane or from a corresponding D2-brane crossing a D6-brane. Yet, the D6-brane is connected to these other branes by dualities. Thus, at least naively it appears that application of supergravity dualities can transform the solutions of [12] into ones in which D6-branes do in fact admit flux from other branes. On the other hand, suppose that these dualities do in fact produce a solution in which the D6-brane admits flux, D4-brane charge flows into the D6-brane at stage (iv), and the IIB solution dual to stage (iv) is a three D5-brane junction. What then will be the IIB story at stages (ii) and (iii) where the branes are not in contact? Recall that it was in fact the flux-expulsion property of our D6-brane that led to the resolution described in section 3. This line of thinking suggests that some subtlety must arise in dualizing the solutions of [12] in this way.

Another avenue that should be explored is to compare the IIB solution discussed here with recent solutions [24, 25, 26] representing M5-brane junctions and other holomorphic curves of M5-branes. Under an appropriate set of operations one should be able to transform these solutions into three-brane junctions and brane-terminating solutions similar to the ones discussed here. Now, the solutions of [24, 25, 26] have certain odd properties of their own. Consider for example [24], which analyzes an intersection two M5-branes in which the two M5-branes should in principle play symmetric roles. Now, the solution found in [24] is valid only in the near-horizon limit of one of the branes. While this does break the explicit symmetry between the branes, the fact that it is supposed to be part of a more complete symmetric solution implies that certain properties of the two branes should be the same. Yet, a close examination of the solution shows that one brane lies at a singularity while the other has a smooth horizon. The second (smooth) brane is the one we are 'near' in the solution and here the spacetime is much like AdS space. Performing a sufficient number of dualities and comparing the result with the solutions of [12] might therefore yield insight into the puzzles associated with both solutions.

In summary, it would appear that two sorts of brane-terminating solutions occur. 
In the first, the termination occurs on a brane that admits flux from other branes. Here the charge of the terminated brane is conserved and flows through the other brane back out to infinity. T-dualizing such solutions leads to further brane-ending solutions or to three-brane junctions. In the second sort, the termination occurs on a brane that for some reason does not capture the proper sort of flux and the charge of the terminating brane evaporates when it contacts the second brane. In this latter case T-duality leads to a solution with charge only at the location of this second brane, the terminating brane having been replaced only with a singularity that carries no charge though it may carry multipole moments under various fields. Whether these two sorts of intersections are connected by dualities remains unclear.

\section{Acknowledgments}

The author would like to thank Neil R. Constable, Ben Craps, Gary Gibbons, Andrés Gomberoff, Jeff Harvey, Clifford Johnson, Emil Martinec, Rob Myers, Amanda Peet, Savdeep Sethi, and Oyvind Tafjord for useful discussions. This work was supported in part by NSF grant PHY97-22362 to Syracuse University, the Alfred P. Sloan foundation, and by funds from Syracuse University.

\section{References}

[1] A. Giveon, M. Porrati, E. Rabinovici, "Target Space Duality in String Theory," Phys. Rept. 24477 (1994), hep-th/9401139.

[2] A. Hanany and E. Witten, "Type IIB Superstrings, BPS Monopoles, and ThreeDimensional Gauge Dynamics," Nucl. Phys. B492, 152 (1997), hep-th/9611230.

[3] U. H. Danielsson, G. Ferretti, and I. R. Klebanov, "Creation of Fundamental Strings by Crossing D-branes," Phys. Rev. Lett. 79 (1997) 1984-1987, hep-th/9705084.

[4] D. Marolf, "Chern-Simons terms and the three notions of charge," hep-th/0006117.

[5] U. Danielsson, G. Ferretti and I. R. Klebanov, "Creation of fundamental strings by crossing D-branes," Phys. Rev. Lett. 79, 1984 (1997), hep-th/9705084.

[6] O. Bergman, M. R. Gaberdiel and G. Lifschytz, "Branes, orientifolds and the creation of elementary strings," Nucl. Phys. B 509, 194 (1998), hep-th/9705130.

[7] S. P. de Alwis, "A note on brane creation," Phys. Lett. B 413, 49 (1997), hep-th/9706142.

[8] P. Ho and Y. Wu, "Brane creation in M(atrix) theory," Phys. Lett. B 420, 43 (1998), hep-th/9708137. 
[9] N. Ohta, T. Shimizu and J. Zhou, "Creation of fundamental string in M(atrix) theory," Phys. Rev. D 57, 2040 (1998), hep-th/9710218.

[10] T. Nakatsu, K. Ohta, T. Yokono and Y. Yoshida, Mod. Phys. Lett. A 13, 293 (1998) hep-th/9711117.

[11] T. Kubota and J. Zhou, "RR charges of D2-branes in group manifold and Hanany-Witten effect," JHEP0012, 030 (2000), hep-th/0010170.

[12] A. Gomberoff and D. Marolf, "Brane transmutation in supergravity," JHEP 0002, 021 (2000), hep-th/9912184.

[13] A. Hashimoto, "Supergravity solutions for localized intersections of branes," JHEP 9901 019 (1999), hep-th/9812159.

[14] N. Itzhaki, A. Tseytlin, and S. Yankielowicz, "Supergravity Solutions for branes localized within branes" Phys. Lett. B432 298-304 (1998), hep-th/9803103.

[15] C. Callan, A. Guijosa, and K. Savvidy, "Baryons and string creation from the fivebrane worldvolume action," Nucl. Phys. B547 (1999) 127-142, hep-th/9810092.

[16] A. Chamblin, R. Emparan and G. W. Gibbons, "Superconducting p-branes and extremal black holes," Phys. Rev. D 58, 084009 (1998) hep-th/9806017.

[17] A. Hashimoto, "The shape of branes pulled by strings," Phys. Rev. D 57, 6441 (1998), hep-th/9711097.

[18] D. Marolf, "Half-branes, singular brane intersections, and Kaluza-Klein reduction," JHEP 0009, 024 (2000), hep-th/0007171.

[19] A. Strominger and C. Vafa, "Microscopic Origin of the Bekenstein-Hawking Entropy," Phys. Lett. B379 99 (1996), hep-th/9601029.

[20] J. Polchinski, String Theory (Cambridge U. Press, 1998).

[21] T. Buscher, "Path Integral Derivation of Quantum Duality in Nonlinear Sigma Models," Phys. Lett. B201 466 (1988); "A Symmetry of the String Background Field Equations," Phys. Lett. B194 59 (1987).

[22] P. Meessen and T. Ortin, "An Sl(2,Z) multiplet of nine-dimensional type II supergravity theories," Nucl. Phys. B541, 195 (1999) hep-th/9806120.

[23] R. Myers, "Dielectric Branes," JHEP 9912, 022 (1999), hep-th/9910053.

[24] A. Fayyazuddin and D. J. Smith, "Warped AdS near-horizon geometry of completely localized intersections of M5-branes," JHEP0010, 023 (2000) hep-th/0006060. 
[25] B. Brinne, A. Fayyazuddin, S. Mukhopadhyay and D. J. Smith, "Supergravity M5branes wrapped on Riemann surfaces and their QFT duals," JHEP0012, 013 (2000) hep-th/0009047.

[26] B. Brinne, A. Fayyazuddin, T. Z. Husain and D. J. Smith, "N = 1 M5-brane geometries," hep-th/0012194. 